\title{
A Modified Blind Equalization Technique Based on a Constant Modulus Algorithm
}

\author{
Jia-Chin Lin and Lin-Shan Lee \\ Department of Electrical Engineering, National Taiwan University, Taipei, Taiwan, R.O.C.
}

\begin{abstract}
A bstract - Improved blind equalization techniques based on a modified constant modulus algorithm (CMA) is proposed in this paper. This algorithm can not only accomplish blind equalization and carrier phase recovery simultaneously, but choose a nonlinear gain with a least-squares algorithm to avoid the gradient noise amplification problem and achieve improved stability and robustness without increasing the computation complexity. It can be switched over to a decision-directed equalization scheme once the error level is reasonably low to obtain higher convergence speed and lower residual intersymbol interference as well. Extensive computer simulation results have verified the analysis and indicated very attractive behavior of the proposed algorithm.
\end{abstract}

\section{INTRODUCTION}

Multipath fading is a major problem with wireless communications. Conventionally, an adaptive equalizer with the aid of a training sequence known to both the transmitting and receiving ends is employed to overcome the effects of multipath fading. However, it has been well known that using such a training sequence to equalize the channel multipath effects is not effective in many applications [1]. As a result, blind equalization techniques have been attracting more and more attention recently. The major advantage of such techniques is that no training sequence is needed to start-up, or restart the system whenever the communication breaks down unpredictably. The technique introduced by Godard [1] has been referenced for such purposes with QAM systems. Many improved techniques have also appeared in the literature $[2,3]$. A modified CMA blind equalization algorithm leading to simultaneous phase recovery and equalization was also proposed by $\mathrm{Oh}$ and $\mathrm{Chin}[3,4]$. In this paper, a further modified CMA algorithm is proposed, which not only leads to simultaneous equalization and phase recovery, but takes the advantage of a least-squares algorithm to achieve better stability, minimal disturbance and improved robustness with noise.

\section{The Constant Modulus Algorithm (CMA) FOR EQUALIZATION}

Consider the baseband model of a digital transmission channel characterized by a finite impulse response
(FIR) filter and an additive white noise. The received signal can be modeled as

$$
x(k)=\sum_{i=0}^{L-1} h(i) a(k-i) e^{j \phi(k)}+n(k)
$$

where $h(i), \quad i=0,1, \cdots, L-1$, are the complex channel tap weights, $L$ is the length of the channel response, $a(k)$ are the complex data symbols, $k$ is the time index, $e^{j \phi(k)}$ is a carrier phase error given by $\phi(k)=2 \pi k \triangle f / R$ introduced by the carrier frequency offset $\triangle f / R$ where $R$ is the symbol rate, and $n(k)$ is the complex white noise. In order to remove the channel distortion, an equalizer with a coefficient vector, $\boldsymbol{W}(k)=\left[w_{0}(k), w_{1}(k), \cdots, w_{N-1}(k)\right]^{T}$ can be employed. The equalizer output is thus

$$
y(k)=\boldsymbol{W}^{\boldsymbol{H}}(k) \boldsymbol{X}(k)
$$

where $\boldsymbol{X}(k)=[x(k), x(k-1), \cdots, x(k-N+1)]^{T}$ is the equalizer input vector. The cost function for conventional CMA to adapt the equalizer tap weights is of the form [1-4]

$$
J(k)=E\left[\left(|y(k)|^{2}-R_{2}\right)^{2}\right]
$$

where $E[\cdot]$ indicates the statistical expectation, $R_{2}$ is a constant depending on the input data symbol $a(k)$,

$$
R_{2}=\frac{E\left\{|a(k)|^{4}\right\}}{E\left\{|a(k)|^{2}\right\}} .
$$

Using a stochastic gradient algorithm to define the update rule, the coefficient vector is adapted by $[1,2]$

$$
\begin{aligned}
W(k+1) & =\boldsymbol{W}(k)-\mu \hat{\nabla} J(k) \\
& =\boldsymbol{W}(k)-\mu \cdot e^{*}(k) \cdot \boldsymbol{X}(k)
\end{aligned}
$$

where $\mu$ is the step-size parameter, and the error signal is $e(k)=y(k)\left(|y(k)|^{2}-R_{2}\right)$ [1-4].

An important problem for the above conventional CMA, however, is that it is phase-blind, because the cost function can only deal with the modulus of the equalizer output. As a result, the equalizer output signal constellation suffers from an arbitrary phase rotation at a rate equal to the carrier frequency offset rate. In 
order to achieve simultaneous blind equalization and carrier phase recovery, the cost function can be rewritten as the following form $[3,4]$ :

$$
J(k)=J_{R}(k)+J_{I}(k)
$$

where $J_{R}(k)$ and $J_{I}(k)$ are the cost functions for real and imaginary parts of the equalizer output $(y(k)=$ $\left.y_{R}(k)+j y_{I}(k)\right)$,

$$
\begin{aligned}
& J_{R}(k)=E\left[\left(y_{R}^{2}(k)-R_{2, R}\right)^{2}\right] \\
& J_{I}(k)=E\left[\left(y_{I}^{2}(k)-R_{2, I}\right)^{2}\right] .
\end{aligned}
$$

Assuming the input data symbols, $a(k)=a_{R}(k)+$ $j a_{I}(k)$, are i.i.d. random variables, $R_{2, R}$ and $R_{2, I}$ can be determined by the real and imaginary parts of the input data symbols respectively,

$$
\begin{aligned}
R_{2, R} & =\frac{E\left\{a_{R}^{4}(k)\right\}}{E\left\{a_{R}^{2}(k)\right\}} \\
R_{2, I} & =\frac{E\left\{a_{I}^{4}(k)\right\}}{E\left\{a_{I}^{2}(k)\right\}}
\end{aligned}
$$

In this way, both the modulus and phase of the signal can be handled simultaneously with a two-dimensional decomposition scheme.

\section{The Proposed Modified CMA Algorithm}

If the decomposed cost functions in Eq. (7) are exploited, the error signal can be rewritten as

$$
\left\{\begin{array}{l}
\tilde{e}_{R}(k)=y_{R}(k)\left(y_{R}^{2}(k)-R_{2, R}\right) \\
\tilde{e}_{I}(k)=y_{I}(k)\left(y_{I}^{2}(k)-R_{2, I}\right) .
\end{array}\right.
$$

Therefore, the modified constant modulus algorithm proposed in this paper can be derived based on the principle of minimal disturbance. It can be considered as the solution to a constrained optimization problem as follows: Given the tap-input vector $\boldsymbol{X}(k)$, determine the next tap-weight vector $W(k+1)$, so as to minimize the squared Euclidean norm of the change in the tapweight vector

$$
\|\delta \boldsymbol{W}(k+1)\|^{2}=\|\boldsymbol{W}(k+1)-\boldsymbol{W}(k)\|^{2}
$$

, subject to the following constraints

$$
\left\{\begin{array}{l}
\operatorname{Re}\left\{\boldsymbol{W}^{H}(k+1) \boldsymbol{X}(k)\right\}^{2}-R_{2, R}=0 \\
\operatorname{Im}\left\{\boldsymbol{W}^{H}(k+1) \boldsymbol{X}(k)\right\}^{2}-R_{2, I}=0 .
\end{array}\right.
$$

To solve this constrained optimization problem, Lagrange multipliers can be used. After some manipula- tion, one can have

$$
\begin{aligned}
& \boldsymbol{W}(k+1)-\boldsymbol{W}(k) \\
= & -\frac{\boldsymbol{X}(k)}{\|\boldsymbol{X}(k)\|^{2}}\left[\frac{\tilde{e}_{R}(k)}{y_{R}(k)\left[y_{R}(k) \pm \sqrt{R_{2, R}}\right]}+j \frac{\tilde{e}_{I}(k)}{y_{I}(k)\left[y_{I}(k) \pm \sqrt{\left.R_{2, I}\right]}\right.}\right]^{*}
\end{aligned}
$$

where $\tilde{e}_{R}(k)$ and $\tilde{e}_{I}(k)$ are defined in Eq. (9).

From a different viewpoint, this problem can also be considered as an underdetermined least-squares estimation problem. Consider the error equation

$$
\epsilon(k)=\left\{ \pm \sqrt{R_{2, R}} \pm j \sqrt{R_{2, I}}\right\}-W^{H}(k+1) X(k)
$$

where $\left\{ \pm \sqrt{R_{2, R}} \pm j \sqrt{R_{2, I}}\right\}$ is the desired average of the equalizer outputs. The problem is then to find the tap-weight vector $\boldsymbol{W}(k+1)$ such that the change in the tap-weight vector given by

$$
\|\delta W(k+1)\|^{2}=\|\boldsymbol{W}(k+1)-W(k)\|^{2}
$$

is minimized, subject to the constraint

$$
\epsilon(k)=0 \text {. }
$$

In this case we can reformulate the error $\epsilon(k)$ as $\epsilon(k)=$ $\overline{\boldsymbol{e}}(k)-\delta \boldsymbol{W}^{H}(k+1) \boldsymbol{X}(k)$, where $\bar{e}(k)=\left\{ \pm \sqrt{R_{2, \boldsymbol{R}}} \pm\right.$ $\left.j \sqrt{R_{2, I}}\right\}-\boldsymbol{W}^{H}(k) \boldsymbol{X}(k)$. Thus, by complex conjugating the both sides of the above equation, we note that the constraint of Eq. (15) is equivalent to

$$
\boldsymbol{X}^{H}(k) \delta \boldsymbol{W}(k+1)=\bar{e}^{*}(k) \text {. }
$$

In this way, we may restate our underdetermined leastsquares estimation problem as finding the minimumnorm solution for the tap-weight vector change $\delta W(k+$ 1) which satisfies Eq. (16). It can be shown that the minimum-norm solution, $\delta W(k+1)=$ $\boldsymbol{X}(k)\left(\boldsymbol{X}^{H}(k) \boldsymbol{X}(k)\right)^{-1} \bar{e}^{*}(k)$, to this underdetermined least-squares estimation problem is exactly the same as that obtained using the method of Lagrange multipliers in Eq. (12). What this means is that given the new input data (at time $k$ ) represented by the input vector $\boldsymbol{X}(k)$, the modified CMA algorithm proposed here updates the tap-weight vector in such a way that the value $\boldsymbol{W}(k+1)$ exhibits a minimum change (in the Euclidean norm sense) with respect to the known value $\boldsymbol{W}(k)$.

Usually, due to stability considerations, the step-size parameter which can be used in the blind equalization algorithms are much smaller than the corresponding values used in the LMS algorithms. Since the stepsize parameter determines the convergence speed, this 
leads to the fact that blind equalizers in general exhibit very slow convergence speed. In order to speed up the convergence process, it has been proposed that the CMA equalization algorithm can be switched to a decision-directed equalization scheme once the error level is reasonably low to form a "dual mode" operation [4]. This approach is also adopted in the modified CMA proposed here. To do this, when we rewrite the update procedure in Eq. (12) as follows,

$\boldsymbol{W}(k+1)-\boldsymbol{W}(k)=-\frac{\mu}{\|\boldsymbol{X}(k)\|^{2}} \boldsymbol{X}(k)\left[\hat{e}_{R}(k)+j \hat{e}_{I}(k)\right]^{*}$

, the error signal, $\left[\hat{e}_{R}(k)+j \hat{e}_{I}(k)\right]$, could thus be approximated as

$$
\begin{aligned}
& \hat{e}_{R}(k)= \begin{cases}\frac{\tilde{e}_{R}(k)}{|y(k)|^{2}}, & y_{R}(k) \notin C_{R} \\
y_{R}(k)-\hat{y}_{R}(k), & y_{R}(k) \in C_{R}\end{cases} \\
& \hat{e}_{I}(k)= \begin{cases}\frac{\tilde{e}_{I}(k)}{|y(k)|^{2}}, & y_{I}(k) \notin C_{I} \\
y_{I}(k)-\hat{y}_{I}(k), & y_{I}(k) \in C_{I}\end{cases}
\end{aligned}
$$

where $C_{R}$ and $C_{I}$ are the confidence zones of real and imaginary parts respectively [4]; $\hat{y}_{R}(k)$ and $\hat{y}_{I}(k)$ are the real and imaginary parts of the decision result of the equalizer output. Initially, the equalization begins when the error is large, the modified CMA proposed above thus operates in the blind mode as described by

$$
\begin{aligned}
& \boldsymbol{W}(k+1)-\underset{\mu}{\boldsymbol{W}}(k) \\
& \cong-\frac{\mu}{\|\boldsymbol{X}(k)\|^{2}|y(k)|^{2}+a} \boldsymbol{X}(k)\left[\tilde{e}_{R}(k)+j \tilde{e}_{I}(k)\right]^{*}
\end{aligned}
$$

where $a$ is a small positive number included to avoid any possible divergence caused by $\|\boldsymbol{X}(k)\|^{2}|y(k)|^{2}=0$. Whenever the output error is low enough, the decisiondirected algorithm with normalized LMS adaptation then takes place in the tracking mode. The update equation is then given by

$\boldsymbol{W}(k+1)-\boldsymbol{W}(k)=-\frac{\mu}{\|\boldsymbol{X}(k)\|^{2}+b} \boldsymbol{X}(k)[y(k)-\hat{y}(k)]^{*}$

where $b$ is a small positive value again and $\hat{y}(k)$ is the decision result of the equalizer output at time $k$. These two modes can be switched over automatically and smoothly according to the magnitude of the equalizer output error without any specific detection mechanis$\mathrm{m}$.

In addition, if we define the step-size parameter as

$$
\hat{\mu}=\frac{\mu}{\|\boldsymbol{X}(k)\|^{2}|y(k)|^{2}+a}
$$

we may view the modified CMA algorithm proposed above as a CMA algorithm with a time-varying stepsize parameter, $\hat{\mu}$. This time-varying step-size parameter can overcome the gradient noise amplification problem usually occurring with ordinary CMA or LMS algorithms whenever the input vector $\boldsymbol{X}(k)$ increases suddenly. Obviously, $\hat{\mu}$ is actually identical to the Kalman filter gain with a fixed positive-definite error covariance matrix to guarantee local stability in the blind-mode of the proposed CMA algorithm. Furthermore, if we replace $y(k)$ in Eq. (21) by $\hat{y}(k)$, i.e., the decision result $\hat{y}(k)$ of the equalizer output is employed as the desired signal in the decision-directed equalization mode, and if $|\hat{y}(k)|^{2}$ may also be replaced by $E\left\{|\hat{y}(k)|^{2}\right\}=1$, we can obtain the step-size parameter for the decision-directed mode of the proposed modified CMA algorithm. Therefore, the equalization of the proposed modified CMA both in the blind mod$\mathrm{e}$ and in the decision-directed mode operates with the same stability as the Kalman filter with a random walk state model.

\section{Simulation Results}

Computer simulation results illustrating the performance of the blind equalization algorithm proposed in this paper are presented in this section. All simulation experiments described in this section employ a complex equalizer of transversal filter structure with 9 tap weights, unless otherwise mentioned, and the equalizers were initialized with the central tap weight set to 1 and others set to zero. The signal to noise ratio (SNR) at the input of the equalizer is defined as

$$
S N R=10 \log _{10} \frac{E\left[|a(k) * h(k)|^{2}\right]}{\sigma_{n}^{2}}(d B)
$$

where $\sigma_{n}^{2}$ is the variance of additive white noise, and * denotes convolution operation. A channel response taken from previous work [3] was used. Shown in Figs. 1(a)-(d) are the signal constellations obtained after an equalizer with conventional CMA, with the modified CMA (MCMA) previously proposed [4], plus dual-mode (MCMA/DM) [4], and the modified CMA proposed here in this paper, respectively, in the case of a carrier frequency offset $\triangle f / R=10^{-4}$ and $S N R=30 d B$. We can see in Fig. 1(a) that the equalizer output signal constellation even spins to form several rings due to lack of carrier frequency lock. On the other hand, Figs. 1(b)-(d) show that the modified CMA proposed in this paper (Fig. 1(d)) can track the offset and cluster the output signal constellation at the 
right position as well as the previous proposed modified CMA with dual mode (MCMA/DM, Fig. 1(c)) better than those without the dual mode (MCMA, Fig. 1(b)). This verified that the modified CMA proposed here can recover both the arbitrary phase rotation caused by the channel characteristics and the spinning phase error due to the carrier frequency offset simultaneously, because it simultaneously performs the envelope equalization and phase error estimation using the twodimensional cost functions, $J_{R}(k)$ and $J_{I}(k)$.

The superiority of normalized LMS (NLMS) over LMS in terms of convergence rate has been illustrated previously [5]. The modified CMA proposed here is derived from a least-squares algorithm, and thus a similar superiority can certainly be achieved as well. This can be demonstrated with the simulation of the convergence behavior and residual ISI as presented below. The residual ISI at the output of the equalizer is defined as follows [6]:

$$
I S I=\frac{\sum_{k}\left[\left|h(k) * w^{*}(k)\right|^{2}\right]-\left|h(k) * w^{*}(k)\right|_{\max }^{2}}{\left|h(k) * w^{*}(k)\right|_{\max }^{2}}
$$

where $\left|h(k) * w^{*}(k)\right|_{\max }$ is the maximum absolute value among all values of $h(k) * w^{*}(k)$. Fig. 2 shows the ensemble average residual ISI obtained from 100 Monte Carlo runs. The results for a channel with additive white Gaussian noise without carrier frequency offset are shown on Fig. 2-(a), while Fig. 2-(b) is for the case with a carrier frequency offset $\triangle f / R=10^{-4}$. The same four algorithms were simulated as in Figs. 1 \& 2 , i.e., the conventional CMA, MCMA, MCMA/DM, and the modified CMA proposed here. We can see in Figs. 3(a) \& (b) that the modified CMA proposed here achieves slightly better convergence speed and residual ISI than the best of the prior arts (MCMA/DM), and the improvement in residual ISI is more significant (up to roughly $2 \mathrm{~dB}$ ) when the carrier frequency offset is present.

In the next simulation experiment, the Gaussian noise is replaced by a double-sided heavy-tailed exponential noise with variance $\sigma_{n^{\prime}}^{2}=0.01$ to model the noise caused by a large delay of echoes [7]. Similar simulation results for convergence speed and residual ISI exactly the same as those in Fig. 2 for the four different algorithms are plotted in Fig. 3. From this figure, we can see that in this situation that the proposed modified CMA performs significantly better than the previous algorithms regardless of whether a carrier frequency offset $\triangle f / R=10^{-4}$ exists or not with at least $2-4 \mathrm{~dB}$ better in residual ISI than the best of the pri- or arts compared here. It is believed that this is because the proposed modified CMA can efficiently avoid the gradient noise amplification due to the heavy-tailed noise.

A further experiment simulating a more realistic pulse jammed multipath fading channel was also performed. In this test a jamming noise $n_{J}(k)$ is also present in addition to the AWGN previously used [8]. The jamming noise waveform, $n_{J}(k)$, is modeled as a complex zero-mean AWGN, with $E\left[\left|n_{J}(k)\right|^{2}\right]=N_{J} / \rho$ for a fraction $\rho$ of the time, and $E\left[\left|n_{J}(k)\right|^{2}\right]=0$ for the remaining fraction $(1-\rho)$ of the time, where $N_{J}$ is the average one-side jammer power spectral density [8]. In the simulation, the AWGN is assumed to have a variance $\sigma_{n}^{2}=0.001, N_{J}=0.01$ and $\rho=0.1$, while the transmitted signal power is normalized to unity. Similar plots for the residual ISI with the different algorithms compared when the carrier frequency offset being absent and present, just as Figs. 2 \& 3, for this channel is shown in Fig. 4. Here significant improvements can be easily found (up to roughly $2-5 \mathrm{~dB}$ ) than the best of the prior arts compared. In particular, in Fig. 4(b) when the carrier frequency offset is present, many serious fluctuations or jumps caused by the impulsive noise and gradient noise amplification effects can be observed for the previous algorithms, while for the modified CMA proposed here such effect is almost negligible. This is because the time-varying step-size parameter can effectively overcome the gradient noise amplification problem. It is therefore concluded that, with Figs. $3 \& 4$, the proposed modified CMA achieves much better robustness and performance stability with respect to various noise characteristics as compared to the previous algorithms, in addition to the higher convergence speed and lower residual ISI.

\section{CONCLUSION}

A modified CMA technique is proposed in this paper. It has been shown that the proposed modified CMA technique can accomplish blind equalization and carrier phase recovery simultaneously. Taking advantage of the least-squares algorithm, the proposed modified CMA exploits a time-varying step-size parameter to handle the non-stability problem and the gradien$\mathrm{t}$ noise amplification problem. The simulation results show that this technique can certainly achieve higher convergence speed, lower residual intersymbol interference, improved stability and robustness, blind equalization and carrier phase recovery simultaneously especially with heavy-tailed noise, impulsive noise. 


\section{REFERENCES}

[1] D. N. Godard, "Self-Recovering Equalization and Carrier Tracking in Two-Dimensional Data Communication Systems," IEEE Trans. Commun., Vol. 28, No. 11, pp. 18671875, Nov. 1980 .

[2] J. R. Treichler and B. G. Agee, "A New Approach to Multipath Correction of Constant Modulus Signals," IEEE Trans. Acoustics, Speech and Signal Processing, Vol. ASSP-31, No. 2, pp. 459-472, April 1983.

[3] K. N. Oh and Y. O. Chin, "Modified Constant Modulus Algorithm: Blind Equalization and Carrier Phase Recovery Algorithm," Proc. ICC'95, pp. 498-502, Seattle, WA, June 1995.

[4] K. N. Oh and Y. O. Chin, "New Blind Equalization Techniques Based on Constant Modulus Algorithm," Proc. Globecomm'95, 1995.

[5] G. C. Goodwin and K. S. Sin, "Adaptive Filter, Prediction, and Control," Englewood Cliffs, N. J., Prentice-Hall, 1984.

[6] O. Shalvi and E. Weinstein, "New Criteria for Blind Deconvolution of Nonminimum Phase Systems (Channels)," IEEE Trans. Inform. Theory, Vol. IT-36, pp. 312-321, Mar. 1990.

[7] D. Ongaro, "Multipath Intermodulation Associated with Operation of FM-FDM Radio Relays in Heavily Built Areas," IEEE Trans. Commun. Technology, Vol. 19, No. 3, June 1971.

[8] I.-K. Chang, G. L. Stuber, and A. M. Bush, "Performance of Diversity Combining Techniques for DS/DPSK Signaling Over a Pulse Jammed Multipath-Fading Channel," IEEE Trans. Commun., Vol.38, No. 10, pp. 1823-1833, Oct. 1990.

[9] S. Haykin, Adaptive Filter Theory, Prentice Hall, Englewood Cliffs.

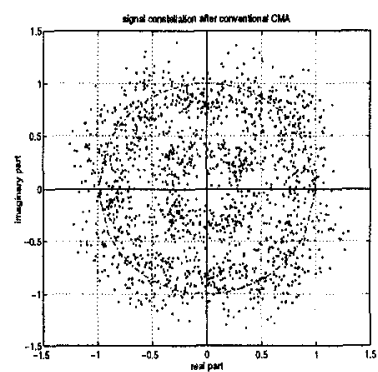

(a)

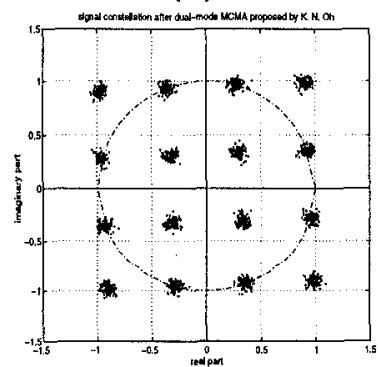

(c)

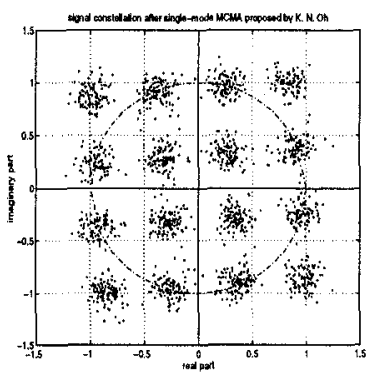

(b)

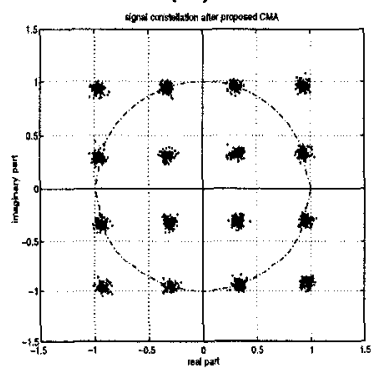

(d)
Fig. 1. The signal constellation after equalization with (a)conventional CMA, (b)modified CMA (MCMA) [4], (c)MCMA plus dual-mode (MCMA/DM) [4], and (d)modified CMA algorithm proposed in this paper, with a carrier frequency offset $\triangle f / R=10^{-4}$.

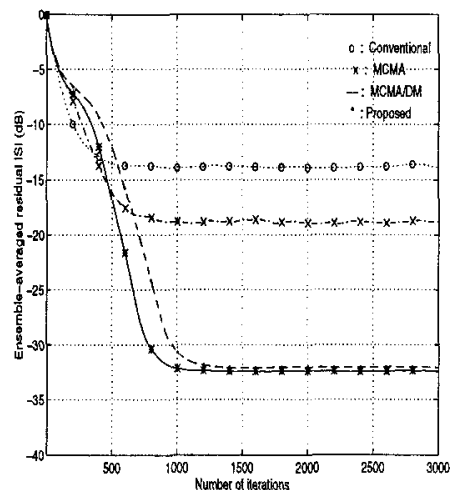

(a)

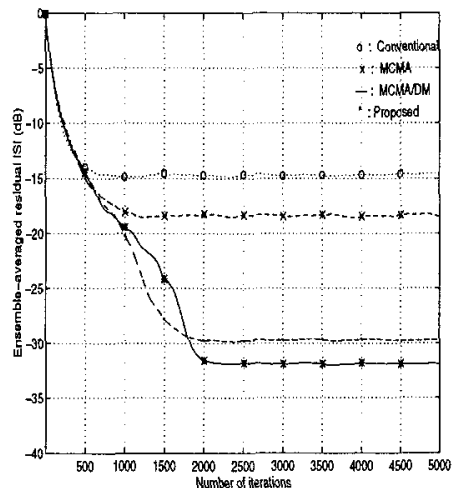

(b)
Fig. 2. The residual ISI (a) with no carrier frequency offset, (b) with a carrier frequency offset $\triangle f / R=10^{-4}$ for the four algorithms compared.

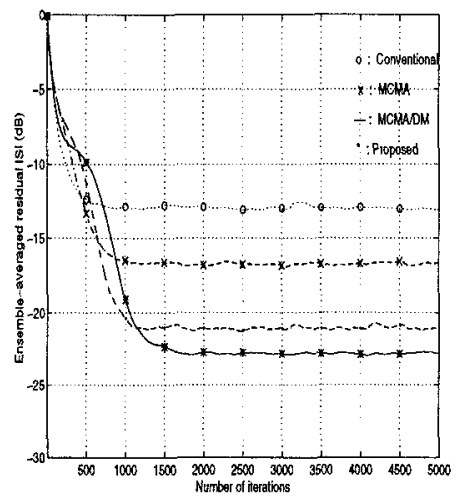

(a)

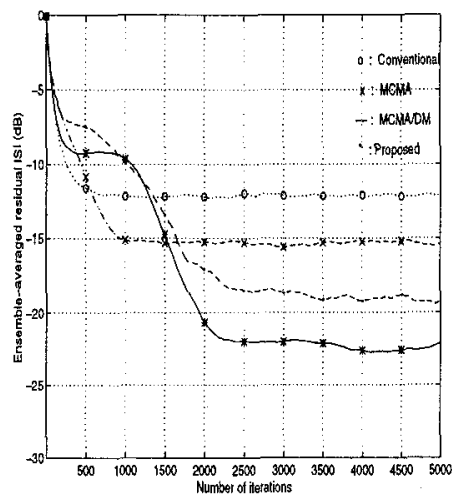

(b)
Fig. 3. The residual ISI (a) with no carrier frequency offset, (b) with a carrier frequency offset $\triangle f / R=10^{-4}$, for additive white exponential noise with variance $\sigma_{n^{\prime}}^{2}=$ 0.01 .

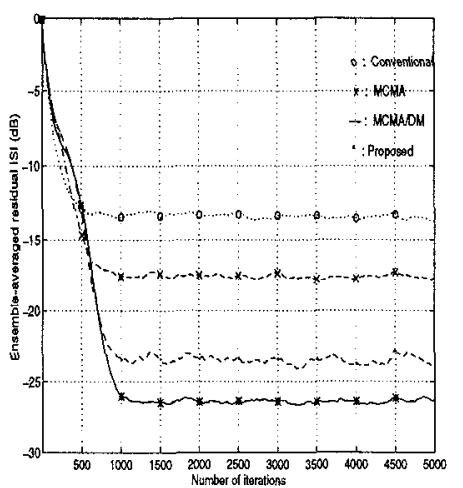

(a)

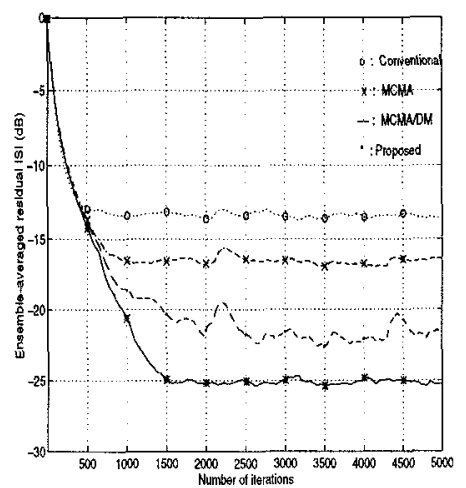

(b)
Fig. 4. The residual ISI (a)with no carrier frequency offset, (b) with a carrier frequency offset $\triangle f / R=10^{-4}$, for additive white Gaussian noise with pulse jams. 